\title{
DISTRIBUTED COMPRESSED SENSING FOR PHOTO-ACOUSTIC IMAGING
}

\author{
K. J. Francis, P. Rajalakshmi, Sumohana S. Channappayya \\ Department of Electrical Engineering, Indian Institute of Technology \\ Hyderabad, India - 502205
}

\begin{abstract}
Photo-Acoustic Tomography (PAT) combines ultrasound resolution and penetration with endogenous optical contrast of tissue. Real-time PAT imaging is limited by the number of parallel data acquisition channels and pulse repetition rate of the laser. Typical photoacoustic signals afford sparse representation. Additionally, PAT transducer configurations exhibit significant intra- and inter- signal correlation. In this work, we formulate photoacoustic signal recovery in the Distributed Compressed Sensing (DCS) framework to exploit this correlation. Reconstruction using the proposed method achieves better image quality than compressed sensing with significantly fewer samples. Through our results, we demonstrate that DCS has the potential to achieve real-time PAT imaging.
\end{abstract}

Index Terms- Photo-Acoustic Tomography, Distributed Compressive Sensing, Joint sparsity

\section{INTRODUCTION}

Photo-Acoustic Tomography (PAT) has gained importance in biomedical imaging in the recent years [1], due to its high spatial resolution and endogenous optical contrast. It is also capable of deep tissue imaging beyond the optical imaging limit [2]. In PAT, a non-ionizing pulsed laser source excites ultrasound waves from the tissue due to thermo-elastic expansion. Thus, PAT effectively combines ultrasound imaging with optical imaging resulting in high contrast, resolution and penetration. PAT has attracted many applications in microscopy, spectroscopy, multiscale imaging, functional and fluorescent imaging and most of them are in the preclinical studies [3]. In many applications, high frequency ultrasound transducers are used to acquire Photo-Acoustic (PA) waves from tissue surface [3] [4]. The spatial sampling resolution of these transducers determines image resolution. High resolution real-time imaging requires a large number of parallel transducers and acquisition channels. To reduce cost, many PAT systems use extensive mechanical scanning and averaging with fewer number of channels. This results in a large number of laser pulse excitations to scan a small area. For scanning deeper tissue, high energy laser pulses are required.

Thanks to IUATC for funding.
Even if one operates within the ANSI limit $\left(20 \mathrm{~mJ} / \mathrm{cm}^{2}\right)$, a large number of laser excitations will adversely affect tissue. Also, low pulse repetition rate in many tunable lasers also increases scanning time. In this scenario, Compressed Sensing (CS) is a potential candidate to help real-time PAT imaging. Under sparsity constraints, CS theory allows us to reduce the number of samples and transducers suggested by the Nyquist rate in temporal and spatial domains. CS is widely used in tomography with applications ranging from Magnetic Resonance Imaging (MRI) [5] to CT and ultrasound imaging. In their seminal paper, Provost et al. introduced CS to PAT [6] where they showed that a sparsely represented PAT image can be reconstructed using CS algorithms. In-vivo experiments with CS has been demonstrated in both time and frequency domains [7] [8]. Several variants of CS in PAT have been studied like reconstruction with partially known support [9], total variation approach [10], fast alternating direct algorithm [11], and the case of recovery from multi-view measurements [12].

In this work, we exploit the rich inter- and intra- signal correlation that exists among localized transducer time series to achieve better compression. To the best of our knowledge, compressed sensing in PAT has not utilized inter signal correlation. We demonstrate how PAT signal recovery can be formulated as a Distributed Compressed Sensing (DCS) problem to exploit this correlation. Specifically, we use a DCS model called Joint Sparsity Model (JSM) - II to model and sense photoacoustic signal. We use the Distributed Compressed Sensing-Simultaneous Orthogonal Matching Pursuit (DCS-SOMP) algorithm [13] for signal recovery.

PAT and DCS models are briefly introduced in Section 2. The problem formulation and the solution are presented in Section 3. We also address the problem of optimal transducer grouping in Section 3. We present and discuss our results in Section 4.

\section{BACKGROUND}

In this section, we discuss both PAT and DCS models to enable us to formulate our DCS-PAT framework. 


\subsection{Photo-Acoustic Imaging}

Pulsed laser with pulse width in the range of few nanosecond can excite Photo-Acoustic (PA) signals in tissue. Under the stress confinement assumption, we can represent the laser pulse as a delta function in time $\delta(t)$, and its spatial component illuminates the tissue surface uniformly [2]. The pressure profile generated by the excitation is given by

$$
P_{0}(\mathbf{r})=\Gamma A(\mathbf{r}),
$$

where $\Gamma=c^{2} \beta / C_{p}$ is a constant called Grüneisen parameter, that quantifies the efficiency of light to sound conversion. $c$ is speed of light, $\beta$ is isobaric volume expansion coefficient, $C_{p}$ is specific heat and $A(\mathbf{r})$ is the optical absorption [2]. In PAT, the PA signals thus generated are acquired by transducers at location $\mathbf{r}$ and time $t$. The propagation of acoustic wave of initial pressure $P_{0}(\mathbf{r})$ to pressure $P(\mathbf{r}, t)$ at the sensing location satisfies the wave equation [14]:

$$
\left(\nabla^{2}-\frac{1}{c^{2}} \frac{\partial^{2}}{\partial t^{2}}\right) P(\mathbf{r}, t)=-P_{0}(\mathbf{r}) \frac{d \delta(t)}{d t} .
$$

The solution for the above equation is given by [1]

$$
P(\mathbf{r}, t)=\frac{\partial}{\partial t}\left[\frac{1}{4 \pi c^{3} t} \int d \mathbf{r}^{\prime} P_{0}\left(\mathbf{r}^{\prime}\right) \delta\left(t-\frac{\left|\mathbf{r}-\mathbf{r}^{\prime}\right|}{c}\right)\right] .
$$

Discretizing the above equation for a circular configuration as in Fig. 1, it can be expressed as an impulse response matrix K [7],

$$
\mathbf{K}_{(i, t),(n, m)}=\frac{1}{2 \pi c} \delta\left(t-\frac{\left|r_{n m}-r_{i}\right|}{c}\right) .
$$

We use a rectangular grid of $N_{x} \times N_{y}$ points, $r_{n m}$ is radius of grid point, $r_{i}$ is $i^{t h}$ transducer location. The time series is measured with $N_{s}$ transducers and $N_{t}$ samples, with sampling interval $T$. In other words, $t=p T$, with $p=1,2, \ldots, N_{t}$ time steps. $n=1,2, \ldots, N_{x}$ and $m=1,2, \ldots, N_{y}$ are $x$ and $y$ axis grid indices respectively. The time series of each transducer can be obtained obtained by

$$
\mathbf{y}_{i, t}=\mathbf{K}_{(i, t),(n, m)} \mathbf{x}_{n, m},
$$

where $\mathbf{x}$ is vectorized initial pressure distribution.

\subsection{Distributed Compressed Sensing (DCS)}

DCS uses multi-signal ensembles that exploit both intra- and inter - signal correlation. Duarte et al. [13] presented three models for joint sparsity of correlated signals - Joint Sparsity Model (JSM) I, II and III. If the signal is not sparse in itself, we can obtain a sparse representation in a different basis $\boldsymbol{\Psi}_{\mathbf{s}}$ (e.g., Fourier, Wavelet etc.) expressed as

$$
\mathbf{y}_{\mathbf{j}}=\Psi_{\mathrm{s}} \theta_{\mathbf{j}}
$$

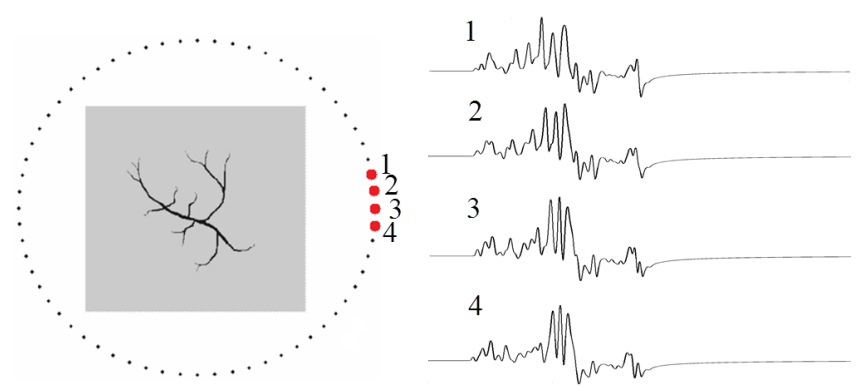

Fig. 1. Circular transducer configuration with initial pressure distribution, computational grid and time series of neighboring transducers showing correlation.

where $\mathbf{y}_{\mathbf{j}}$ are correlated signals, $j \in\{1,2 \ldots, J\}, \theta_{\mathbf{j}}$ are supported on $\boldsymbol{\Omega} \subset\left\{1,2,3 \ldots, N_{t}\right\}$ with $|\boldsymbol{\Omega}|=S$, where $S$ is signal sparsity. Sensing of a group of $J$ signals is given by

$$
v_{\mathbf{j}}=\Phi_{\mathbf{j}} \mathbf{y}_{\mathbf{j}}
$$

The sensing matrices $\boldsymbol{\Phi}_{\mathbf{j}}$ are different for individual signals and are of size $M \times N_{t}$, where $M=c S$ with oversampling rate $c$. For groups with large number of sensors, $c=\frac{S+1}{S}$ points to the fact that that number of measurement $(M)$ required to recover the signals is close to $S$, the number of nonsparse terms in the signal.

\section{DCS FOR PAT}

\subsection{Problem formulation}

We first empirically demonstrate inter-signal correlation in a circular transducer configuration for PAT. Fig. 1 shows representative time series of 4 neighboring transducers sampling a generic signal. It is clear from the figure that the measurements made at neighboring transducers are highly correlated. The key observation from these measurements is that the JSM-II model proposed in [13] nicely fits in this setting where the signals are correlated and have the same support set. We would like to recall here that the JSM-II model considers a scenario where multiple sensors acquire the same signal but with different phase shifts and attenuation due to propagation in a medium.

Time series of individual transducers, $\mathbf{y}_{\mathbf{j}}=\mathbf{y}_{i, t}$ are randomly sensed using sensing matrices $\boldsymbol{\Phi}_{\mathbf{j}}$. With the knowledge of the number of non-zero terms in the signal $S$, we can achieve optimal compression by choosing the number of measurements $M$ so as to minimize recovery error. The choice of $M$ also depends on how many correlated signals are grouped together during recovery. Once we find this $M$, our aim is to find how to recover the signal exactly. 


\subsection{Solution}

Having formulated the signal compression problem in the DCS framework, it follows naturally that the solution to JSM-II applies to this formulation as well. For completeness, we outline the DCS-SOMP in Algorithm 1 with appropriate references to our formulation. The readers are referred to [13] for a detailed description. Once the signal is recovered, the

Step 1 Initialize:

$\Upsilon_{\mathbf{j}}=\Phi_{\mathbf{j}} \Psi_{\mathrm{s}}$

Iteration $l=1$,

Orthogonalized coefficient $\hat{\beta}_{j}=0$,

Support set $\hat{\boldsymbol{\Omega}}=[$ ]

Residue $\mathbf{r}_{j, 0}=v_{j}$

Step 2 Support selection

$n_{l}=\underset{n=1,2, \ldots, N}{\operatorname{argmin}} \sum_{j=1}^{J} \frac{\left|\left\langle\mathbf{r}_{\mathbf{j}, 1-\mathbf{1}}, \boldsymbol{\Upsilon}_{j, n}\right\rangle\right|}{\left\|\mathbf{\Upsilon}_{j, n}\right\|_{2}}$

$\hat{\mathbf{\Omega}}=\left[\hat{\mathbf{\Omega}} n_{l}\right]$

Step 3 Orthogonalize basis

$\gamma_{j, l}=\boldsymbol{\Upsilon}_{n, n_{l}}-\sum_{t=0}^{l-1} \frac{\left\langle\boldsymbol{\Upsilon}_{j, n_{l},}, \gamma_{j, t}\right\rangle}{\left\|\mathbf{r}_{j, t}\right\|_{2}^{2}} \gamma_{j, t}$

Step 4 Update coefficient

$\hat{\beta}_{\mathbf{j}}(l)=\frac{\left\langle\mathbf{r}_{j, l-1}, \gamma_{j, l}\right\rangle}{\left\|\gamma_{j, l}\right\|_{2}^{2}}$

$\mathbf{r}_{j, l}=\mathbf{r}_{j, l-1}-\frac{\left\langle\mathbf{r}_{j, l-1}, \gamma_{j, l}\right\rangle}{\left\|\gamma_{j, l}\right\|_{2}^{2}} \gamma_{j, l}$

Step 5 Convergence check

$\left\|\mathbf{r}_{j, l}\right\|_{2}>\epsilon\left\|v_{j}\right\|_{2}$

then $l=l+1$ continue to step 2, otherwise

convergence satisfied.

Step 6 De-orthogonalize

Apply QR factorization to mutilated basis $\Upsilon_{\hat{\mathbf{\Omega}}}$

$$
\begin{aligned}
\boldsymbol{\Upsilon}_{j, \hat{\mathbf{\Omega}}}=\mathbf{Q}_{j} \mathbf{R}_{j} \text { then } \theta_{j, \hat{\Omega}} & =\mathbf{R}_{j}^{-1} \hat{\beta}_{j} \\
\hat{\mathbf{y}}_{j} & =\mathbf{\Psi}_{\mathbf{s}} \hat{\theta}_{j}
\end{aligned}
$$

Vectorize the individual $\hat{\mathbf{y}}_{j}$ to get reconstructed measurement $\hat{\mathbf{y}}$

Algorithm 1: DCS-SOMP Algorithm

image can be reconstructed using any standard reconstruction algorithm used in PAT. We use model-based method to reconstruct the image [7].

\subsection{Optimal grouping and oversampling strategy}

The proposed formulation poses a couple of questions. Given a set of $N_{s}$ transducers, what is the optimal number of transducers $J$ to be grouped, so as to get maximum signal compression? Secondly, for a particular grouping, what should be the minimum oversampling rate $c$ ? Consider the transducer configuration presented in Fig. 1. The relation between oversampling $c$ required and the number of transducers grouped for perfect reconstruction of photoacoustic signal is shown in Fig. 2. To test correlation among signals, 128 transducers are employed and a grouping of $J=2,4,8,16$ and 32 are con- sidered. Number of measurements are varied from 10 to 50 . Most of the time series have a sparsity between 21 and 25 . It is evident from the graph that as more and more transducers are grouped in JSM, the number of samples required to get exact reconstruction reduces and approaches sparsity $(S)$ for large value of $J . J=1$ does not exploit correlation and hence it is equivalent to compressed sensing case. From $J=2$ to 16 , samples required for exact reconstruction decreases and become equal to sparsity rate $(S=21)$ at $J=16$. It is interesting to note that at $J=32$ and 64 the oversampling rate increases due to decrease in correlation.

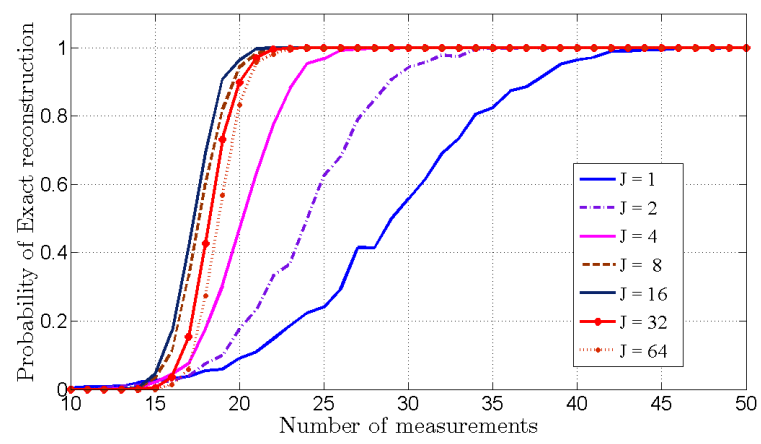

Fig. 2. Probability of exact reconstruction vs. measurement for different value of transducer grouping $(J)$, averaged over 1000 iterations.

\section{RESULTS AND DISCUSSION}

We conducted numerical experiments to prove the efficiency of the proposed algorithm. PAT forward operation is performed using (5), which maps image $\mathrm{x}$ to transducer's time series $\mathbf{y}$. Both $\mathbf{x}$ and $\mathbf{y}$ are vectorized and $\mathbf{K}$ is obtained using (4). In all simulations, computational grid size is taken as $20 \mathrm{~mm} \times 20 \mathrm{~mm}$ with a resolution of $64 \times 64$. Transducers are considered as point detectors and are arranged in a circular configuration as shown in Fig. 1 with a radius of $15 \mathrm{~mm}$ from the center of grid.

In this setting, we found that correlation between transducer time series increases as number of transducers increase, since distance between them reduces. However, optimal grouping must be performed to exploit the correlation as shown in Section 3.3. Gaussian and Bernoulli matrices are known to satisfy Restricted Isometric Property [15] and can also be implemented in hardware [16]. We used these matrices in our experiments for random sensing (7). PA signals are then recovered using Algorithm 1. Once the PA signals are recovered, the image is reconstructed. For image recovery using compressed sensing we used $l_{1}$ minimization [6]. Figs. 3 and 4 shows reconstructed image using CS and DCS algorithm with $N_{s}$ transducers and $N_{t}$ samples. We used Vein and Shepp Logan phantoms due to their dissimilar sparsity 


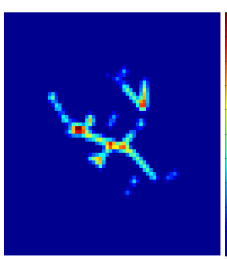

(a) Initial pressure

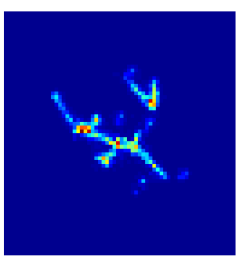

(c) CS $48 \times 24$

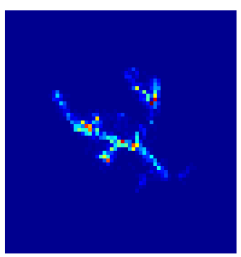

(b) CS $32 \times 24$

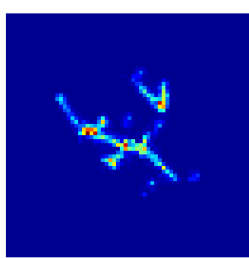

(e) DCS $48 \times 24, J=4$
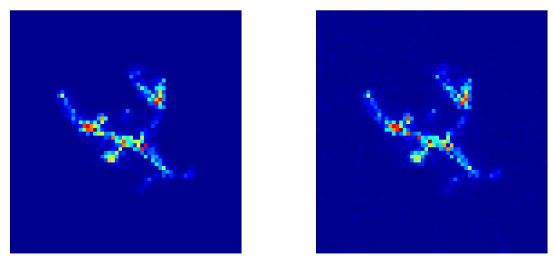

(d) $\operatorname{DCS} 32 \times 24, J=4($ f) $\operatorname{DCS} 32 \times 16, J=8$
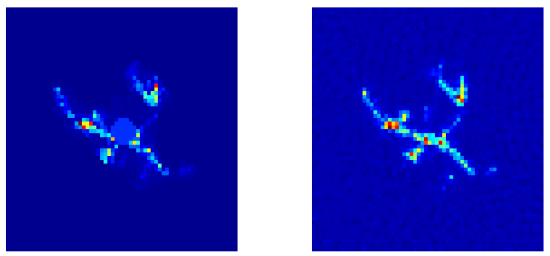

(d) CS $48 \times 16$

(e) DCS $48 \times 16, J=8$

Fig. 3. Reconstruction of vein phantom using CS and DCS with $N_{s}$ number of transducer and $N_{t}$ samples.

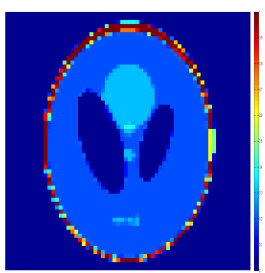

(a) Initial pressure

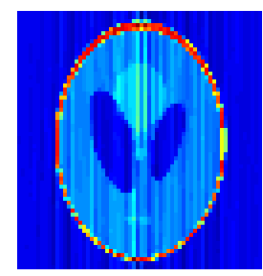

(d) CS $64 \times 32$

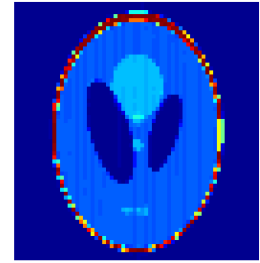

(b) DCS $64 \times 32$

Fig. 4. Reconstruction of Shepp Logan Phantom using CS and DCS with $N_{s}$ number transducer and $N_{t}$ samples.

\begin{tabular}{|c|c|c|c|}
\hline Method $\left(N_{s} \times N_{t}\right)$ & MSE & PSNR & SSIM \\
\hline \multicolumn{4}{|c|}{ Vein Phantom } \\
\hline $\mathrm{CS} 32 \times 24$ & 0.0024 & 26.1416 & 0.8964 \\
\hline DCS $32 \times 24$ & 0.0017 & 27.7892 & 0.9180 \\
\hline DCS $32 \times 16$ & 0.0016 & 27.8914 & 0.9175 \\
\hline $\mathrm{CS} 48 \times 32$ & 08 & 30.8277 & 0.9625 \\
\hline DCS $48 \times 24$ & 0.0008 & 30.8277 & 0.9625 \\
\hline CS $48 \times 16$ & 0.0029 & 25.3257 & 0.8634 \\
\hline DCS $48 \times 16$ & 0.0012 & 29.3295 & 0.8957 \\
\hline \multicolumn{4}{|c|}{ Shepp Logan Phantom } \\
\hline $\mathrm{CS} 64 \times 32$ & 0.0029 & 25.3257 & 0.8634 \\
\hline DCS $64 \times 32$ & 0.0012 & 29.3295 & 0.8957 \\
\hline
\end{tabular}

Table 1. Comparison of reconstructed image quality, using Compressed Sensing (CS) and Distributed Compressed Sensing (DCS) method with $N_{s}$ transducers and $N_{t}$ time steps

properties. For Shepp Logan phantom we used Numerical Derivative as sparsifying matrix.

The image quality obtained with CS and DCS is evaluated using Mean Square Error (MSE), Peak Signal to Noise Ratio
(PSNR) and the Structural Similarity (SSIM) index in Table 1. This clearly shown that DCS achieves a better image quality than CS with much fewer samples for a fixed number of transducers. As hypothesized, we were able to exploit inter signal correlation in photo-acoustic signals and were able to reconstruct images with fewer samples using the DCS-SOMP algorithm.

\section{CONCLUSIONS AND FUTURE WORKS}

We have empirically demonstrated how PAT reconstruction can be formulated in the DCS framework, to reduce number of samples used for reconstruction. Using JSM - II, the inter signal correlation between transducer time series is exploited for better compression. Our results show that we can achieve better image quality with far fewer samples than conventional compressed sensing used in PAT, which depend only on sparsity of image. As future work, we intend to formulate a single step reconstruction algorithm utilizing DCS, which can exploit inter and intra signal correlation and directly recovers the image. We also plan to test the framework with real photoacoustic signals generated from tissue. 


\section{REFERENCES}

[1] Minghua Xu and Lihong V Wang, "Photoacoustic imaging in biomedicine," Review of scientific instruments, vol. 77, no. 4, pp. 041101, 2006.

[2] Lihong V Wang, Photoacoustic imaging and spectroscopy, CRC press, 2009.

[3] Lihong Wang, "Photo acoustic tomography," Scholarpedia, vol. 9, no. 2, pp. 10278, 2014.

[4] John Gamelin, Anastasios Maurudis, Andres Aguirre, Fei Huang, Puyun Guo, Lihong V Wang, and Quing Zhu, "A real-time photoacoustic tomography system for small animals," Optics express, vol. 17, no. 13, pp. 10489-10498, 2009.

[5] Michael Lustig, David L Donoho, Juan M Santos, and John M Pauly, "Compressed sensing mri," Signal Processing Magazine, IEEE, vol. 25, no. 2, pp. 72-82, 2008.

[6] Jean Provost and Frederic Lesage, "The application of compressed sensing for photo-acoustic tomography," Medical Imaging, IEEE Transactions on, vol. 28, no. 4, pp. 585-594, 2009.

[7] Jing Meng, Lihong V Wang, Dong Liang, and Liang Song, "In vivo optical-resolution photoacoustic computed tomography with compressed sensing," Optics letters, vol. 37, no. 22, pp. 4573-4575, 2012.

[8] Zijian Guo, Changhui Li, Liang Song, and Lihong V Wang, "Compressed sensing in photoacoustic tomography in vivo," Journal of Biomedical Optics, vol. 15, no. 2, pp. 021311-021311, 2010.

[9] Jing Meng, Lihong V Wang, Leslie Ying, Dong Liang, and Liang Song, "Compressed-sensing photoacoustic computed tomography in vivo with partially known support," Optics Express, vol. 20, no. 15, pp. 16510-16523, 2012.

[10] Yan Zhang, Yuanyuan Wang, and Chen Zhang, "Total variation based gradient descent algorithm for sparseview photoacoustic image reconstruction," Ultrasonics, vol. 52, no. 8, pp. 1046-1055, 2012.

[11] Xueyan Liu, Dong Peng, Wei Guo, Xibo Ma, Xin Yang, and Jie Tian, "Compressed sensing photoacoustic imaging based on fast alternating direction algorithm," Journal of Biomedical Imaging, vol. 2012, pp. 12, 2012.

[12] NZ Feng, MJ Sun, LY Ma, and ZH Wu, "Compressive sensing photoacoustic imaging based on multi-view error gradient fusion," IET optoelectronics, vol. 6, no. 2, pp. 117-120, 2012.
[13] Marco F Duarte, Shriram Sarvotham, Dror Baron, Michael B Wakin, and Richard G Baraniuk, "Distributed compressed sensing of jointly sparse signals," in Asilomar Conf. Signals, Sys., Comput, 2005, pp. 1537-1541.

[14] Minghua Xu and Lihong V Wang, "Universal backprojection algorithm for photoacoustic computed tomography," Physical Review E, vol. 71, no. 1, pp. 016706, 2005.

[15] Emmanuel J Candes, "The restricted isometry property and its implications for compressed sensing," Comptes Rendus Mathematique, vol. 346, no. 9, pp. 589-592, 2008.

[16] Moshe Mishali, Yonina C Eldar, Oleg Dounaevsky, and Eli Shoshan, "Xampling: Analog to digital at subnyquist rates," IET circuits, devices \& systems, vol. 5, no. 1 , pp. 8-20, 2011. 